\title{
ChemComm
}

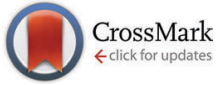

Cite this: Chem. Commun., 2016, 52,7470

Received 16th April 2016, Accepted 12th May 2016

DOI: $10.1039 / c 6 c c 03199 d$

www.rsc.org/chemcomm

\section{A metal sulfide photocatalyst composed of ubiquitous elements for solar hydrogen production $\dagger$}

\author{
Y. Shiga, ${ }^{a}$ N. Umezawa, ${ }^{* b}$ N. Srinivasan, ${ }^{a}$ S. Koyasu, ${ }^{a}$ E. Sakai ${ }^{a}$ and M. Miyauchi ${ }^{a}$
}

\begin{abstract}
A visible-light-sensitive tin sulfide photocatalyst was designed based on a ubiquitous element strategy and density functional theory (DFT) calculations. Computational analysis suggested that tin monosulfide (SnS) would be more efficient than $\mathrm{SnS}_{2}$ as a photocathode for hydrogen production because of the low ionization potential and weak ionic character of SnS. To test this experimentally, nanoparticles of SnS were loaded onto a mesoporous electrode using a wet chemical method, and the bandgap of the synthesized SnS quantum dots was found to be tunable by adjusting the number of successive ionic layer adsorption and reaction (SILAR) cycles, which controls the magnitude of the quantum confinement effect. Efficient hydrogen production was achieved when the bandgap of SnS was wider than that of the bulk form.
\end{abstract}

For efficient photocatalytic solar water splitting, materials with visible-light harvesting ability and electrons and/or holes with high mobilities for promoting charge separation are required. Several efficient metal oxide semiconductor photocatalysts, which typically have $\mathrm{d}^{0}$ or $\mathrm{d}^{10}$ metal orbital configurations, have been reported to date. ${ }^{1-3}$ Most of these metal oxides have a wide bandgap, as their valence band consists of a deep oxygen $2 \mathrm{p}$ orbital, and hole carriers have a relatively heavy effective mass due to the localized nature of the oxygen $2 \mathrm{p}$ state. In contrast to metal oxides, metal sulfides generally have a shallow valence band ${ }^{4}$ and exhibit a clear quantum size effect due to their small effective mass. ${ }^{5}$ These properties contribute to the visible-light harvesting ability and high charge mobility of metal sulfides. ${ }^{6,7}$ Several previous studies have examined the photoenergy conversion of metal sulfide quantum dots (QDs), including $\mathrm{CdS}, \mathrm{CuInS}_{2}$, and PbS QDs, ${ }^{8-12}$ as the bandgap, and conduction and valence band potentials of the materials are tunable based

\footnotetext{
${ }^{a}$ School of Materials and Chemical Technology, Tokyo Institute of Technology,

2-12-1 Ookayama, Meguro, Tokyo 152-8552, Japan.

E-mail:mmiyauchi@ceram.titech.ac.jp

${ }^{b}$ International Center for Materials Nanoarchitectonics, National Institute for

Materials Science (NIMS), 1-1 Namiki, Tsukuba, Ibaraki 305-0044, Japan.

E-mail: Umezawa.Naoto@nims.go.jp

$\dagger$ Electronic supplementary information (ESI) available. See DOI: 10.1039/c6cc03199d
}

on their particle size. ${ }^{13,14}$ However, because these metal sulfides are composed of toxic and/or rare elements, the design of metal sulfide semiconductors composed of safe and abundant elements is required for use in practical applications. This approach is consistent with the "ubiquitous element strategy" that has recently been proposed for materials used in industrial activities and has attracted considerable attention towards a safe and sustainable society. ${ }^{15-17}$ Previous studies reported the efficient materials for solar-to-energy conversion with ubiquitous earth-abundant elements, like carbon nitride and boron nitride. ${ }^{18-20}$

Among the various elements in the periodic table, we have focused on group IV-VI chalcogenides due to their excellent optoelectronic properties and high charge mobilities. ${ }^{21}$ In particular, tin sulfides, which have unique electrical characteristics, various valence numbers, and sensitivity to a wide range of visible light, are attractive materials for use in electronic semiconductor devices and thin-film solar cells. ${ }^{22,23}$ Tin sulfides have two chemical compositions: tin monosulfide ( $\mathrm{SnS}$ ) and tin disulfide $\left(\mathrm{SnS}_{2}\right)$. Herein, the electronic structures of $\mathrm{SnS}$ and $\mathrm{SnS}_{2}$ were elucidated based on density functional theory (DFT) calculations, and the semiconductor characteristics of these compounds were also evaluated. In addition, $\mathrm{SnS}$ and $\mathrm{SnS}_{2}$ QDs were synthesized by a facile wet chemical method. For the synthesis, a mesoporous $\mathrm{TiO}_{2}$ electrode was used as the support, since minute pores are helpful for fixing QDs on the electrode support. The deposition of nanometer-scale metal sulfide QDs is expected to induce the quantum confinement effect and improve the photochemical properties of the material compared to the bulk form. The photoelectrochemical properties and photocatalytic hydrogen production activities of the tin sulfide QD-modified electrodes were also evaluated. Further, the effect of a thin layer of amorphous $\mathrm{SiO}_{2}$ on the stability of the tin sulfide QDs was examined under visible-light irradiation.

Fig. 1 shows the calculated band diagrams of $\mathrm{SnS}$ and $\mathrm{SnS}_{2}$. The bandgap of SnS was narrower than that of $\mathrm{SnS}_{2}$, and both the ionization potential and electron affinity of SnS were smaller than those of $\mathrm{SnS}_{2}$. The conduction band potential of $\mathrm{SnS}$ is higher than the potential for hydrogen production. Band dispersion and 


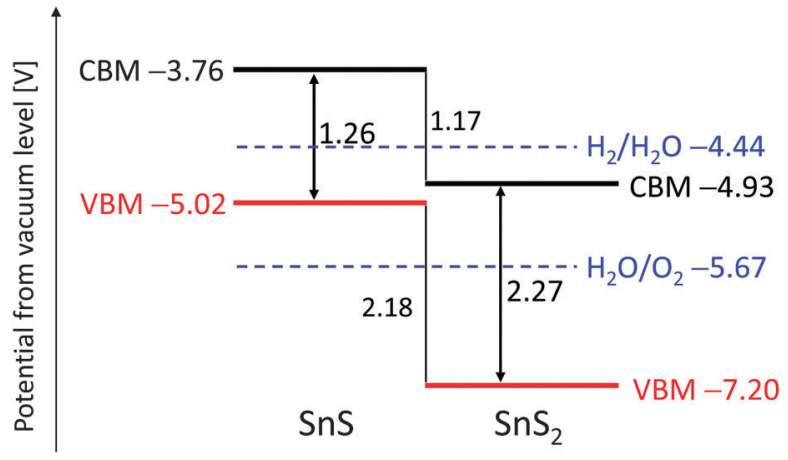

Fig. 1 Band diagram of $\mathrm{SnS}$ and $\mathrm{SnS}_{2}$ based on DFT calculations.

density of states were also calculated for $\mathrm{SnS}$ and $\mathrm{SnS}_{2}$ and showed that both materials are indirect transition semiconductors, as shown in the ESI $\dagger$ (Fig. S1), which was consistent with the previous reports. ${ }^{24-28}$ Notably, the valence band of SnS consisted of Sn-5s and S-3p hybrid orbitals, suggesting that SnS would function as a p-type semiconductor. Burton et al. $^{22,23}$ also reported a similar band structure for tin sulfides and suggested that SnS would be a suitable semiconductor for thin-film solar cells. Based on our DFT calculations, SnS was also predicted to be potentially useful as a photocathode for hydrogen production, although SnS is unable to evolve oxygen from water. In contrast, $\mathrm{SnS}_{2}$ would not produce hydrogen from water because of its low conduction band potential.

We loaded SnS QDs onto mesoporous $\mathrm{TiO}_{2}$ electrodes using the successive ionic layer adsorption and reaction (SILAR) method, ${ }^{29,30}$ and $\mathrm{SnS}_{2}$ particles were loaded onto $\mathrm{TiO}_{2}$ electrodes using a hydrothermal reaction. ${ }^{31}$ Detailed experimental procedures are described in the ESI. $\uparrow$ The crystal phases of SnS and $\mathrm{SnS}_{2}$ were Herzenbergite (ICDD No. 00-039-0354) and Berndtite (ICDD No. 00-023-0677), respectively, as determined by X-ray diffraction patterns (XRD, Fig. 2a). Analysis of X-ray photoelectron spectroscopy (XPS) spectra for the Sn-3d and S-2p orbitals (Fig. 2b and c) revealed the chemical shift, owing to differences in the valence numbers of $\mathrm{SnS}$ and $\mathrm{SnS}_{2} \cdot{ }^{32}$ In addition, chemical composition analysis by inductively coupled plasma (ICP) spectroscopy revealed that the atomic ratios of $\mathrm{S} / \mathrm{Sn}$ in $\mathrm{SnS}$ and $\mathrm{SnS}_{2}$ were nearly 1 and 2, respectively. The particle sizes of SnS (10 SILAR cycles) and $\mathrm{SnS}_{2}\left(150{ }^{\circ} \mathrm{C}\right.$ hydrothermal temperature)

\section{(a)}

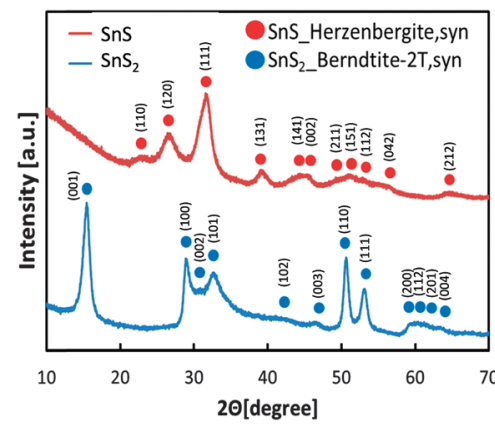

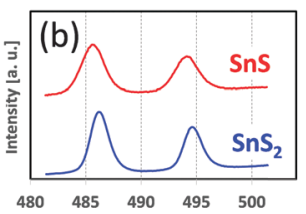
Binding Energy [eV]

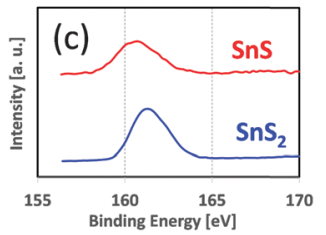

Fig. 2 XRD diffraction patterns of $\mathrm{SnS}$ and $\mathrm{SnS}_{2}$ powder (a), and XPS spectra for Sn-3d (b) and S-2p orbitals (c).
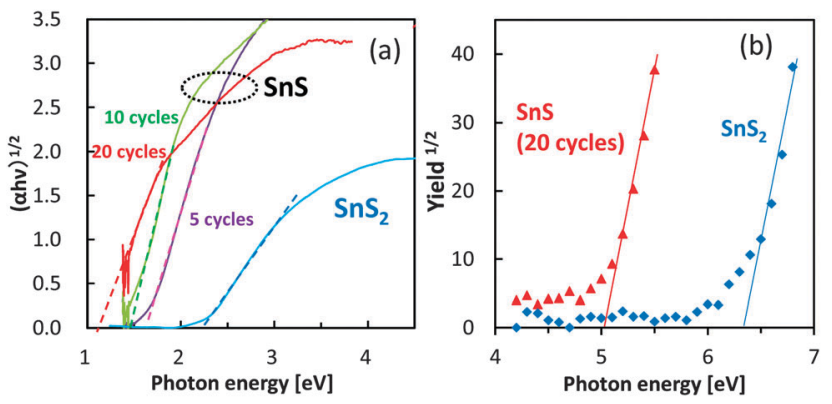

Fig. 3 UV-Vis spectra (a) and ultraviolet photoelectron spectra (b) of SnS electrodes. The SnS electrodes in panel (a) were prepared using the SILAR method with 5, 10, and 20 cycles.

were determined to be approximately $10 \mathrm{~nm}$, based on TEM observation (Fig. S2, ESI $\dagger$ ).

The optical absorption properties of $\mathrm{SnS}$ and $\mathrm{SnS}_{2}$ are shown in Fig. 3a. The bandgap of SnS appeared to be narrower than that of $\mathrm{SnS}_{2}$, a finding that is consistent with the DFT calculations. Notably, the bandgap of SnS was dependent on the number of SILAR cycles. Specifically, with increasing number of SILAR cycles, the absorption of the SnS particles shifted towards lower energy, a property that was attributed to the quantum confinement effect. The colour of SnS changed from orange to brown with the increasing number of SILAR cycles, whereas the $\mathrm{SnS}_{2}$ electrode was yellow in colour (Fig. S4, ESI†).

The ultraviolet photoelectron spectra of $\mathrm{SnS}$ and $\mathrm{SnS}_{2}$ were next determined to examine the valence band potential of these sulfides (Fig. 3b). The ionization potential of SnS was lesser than that of $\mathrm{SnS}_{2}$, suggesting that $\mathrm{SnS}$ had a shallower valence band. The observed valence band positions were also consistent with the DFT calculations.

The relationship between photocurrent densities and bandgap values for the $\mathrm{SnS}$ and $\mathrm{SnS}_{2}$ electrodes was also investigated under chopped irradiation by a solar simulator without bias application (Fig. 4a). A small photocurrent was detected from the bare $\mathrm{TiO}_{2}$ electrode used as the baseline control (Fig. 4a, dashed line) and was attributed to the small amount of UV light that passed through the optical UV cut-off filter. Photocurrent generation by $\mathrm{SnS}_{2}$ was similar to that of bare $\mathrm{TiO}_{2}$, suggesting that the hydrogen generation rate in the $\mathrm{SnS}_{2}$ system was limited. In contrast, photocurrent by the SnS electrodes was significant, and the optimum bandgap value for the maximal photocurrent was approximately $1.4 \mathrm{eV}$, which was wider than the bulk bandgap value. Photon energy with $1.4 \mathrm{eV}$ matches with the solar light spectrum. If the bandgap is wider than $1.5 \mathrm{eV}$, the number of electron-hole pairs are reduced due to the limited optical absorption. On the other hand, as the bandgap narrows with increasing number of SILAR cycles, the reduction potential of SnS was limited for hydrogen production. Further, as the bandgap narrows with increasing number of SILAR cycles, the $\mathrm{TiO}_{2}$ electrode surface becomes overloaded with $\mathrm{SnS}$ as free particles, which do not have contact with the mesoporous support. Under this condition, electron transport to the Pt electrode is limited. Also, overloaded SnS particles prevent the diffusion of electrolyte agent to the active SnS particles attached on $\mathrm{TiO}_{2}$. 

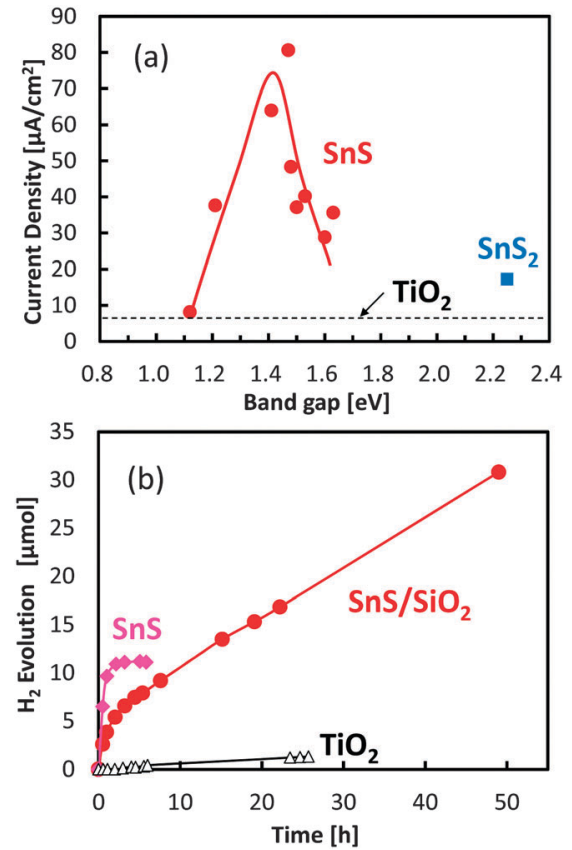

Fig. 4 Photocurrent density under an AM 1.5 M solar simulator versus the bandgap energy of the semiconductor (a), and photocatalytic hydrogen evolution under visible-light irradiation (b). In both (a) and (b), visible light was irradiated through a UV cut-off filter, and a bias potential was not applied on the electrode. For the measurement of photocurrent densities, the $\mathrm{Pt}$ wire and the $\mathrm{Ag} / \mathrm{AgCl}$ electrode were used as counter and reference electrodes, respectively. For the analysis of hydrogen production, a thin $\mathrm{Pt}$ layer was inserted between $\mathrm{FTO}$ and the mesoporous $\mathrm{TiO}_{2}$ layer using a sputtering method as a cocatalyst for hydrogen production. The electrolyte solution contained $\mathrm{Na}_{2} \mathrm{~S}$ and $\mathrm{Na}_{2} \mathrm{SO}_{3}$ as sacrificial agents for the oxidation reaction.

Therefore, the bandgap value of $1.4 \mathrm{eV}$ for $\mathrm{SnS}$ determined in the present study appears to be optimal for solar hydrogen production. The results of control experiments, such as that using the $\mathrm{SnS} / \mathrm{TiO}_{2}$ electrode without the Pt layer, are shown in our ESI $\dagger$ (Fig. S6), but the hydrogen production from these electrodes was negligible.

Photocatalytic hydrogen production by SnS with an optimized bandgap was further examined under visible-light irradiation without the application of a bias potential (Fig. $4 \mathrm{~b}$ ). SnS produced hydrogen under photon flux, and the quantum efficiency was calculated to be $0.31 \%$, which was determined by the number of reacted electrons versus the absorbed photon numbers (see our ESI $\dagger$ ). However, hydrogen production ceased after several hours of irradiation, owing to the photo-corrosion of SnS. In an attempt to avoid the photo-corrosion of SnS, a thin layer of amorphous silica $\left(\mathrm{SiO}_{2}\right)$ with a thickness of less than $2 \mathrm{~nm}$ (Fig. S7, ESI $\dagger$ ) was coated on the SnS electrode. Although the initial hydrogen production rate of the $\mathrm{SiO}_{2}$-coated $\mathrm{SnS}$ electrode was lower than that of the uncoated electrode, the stability of the SnS photocatalyst was significantly improved by the thin $\mathrm{SiO}_{2}$ coating. The calculated turnover number for the $\mathrm{SiO}_{2}$-coated $\mathrm{SnS}$ electrode was 4.2 after $50 \mathrm{~h}$ of visible-light irradiation, and hydrogen production continued to be produced even after $50 \mathrm{~h}$. The improved stability is attributed to passivation by the aqueous electrolyte and/or tunnelling of charge carriers through the nanometer-scale $\mathrm{SiO}_{2}$ layer. ${ }^{33}$ The brown colour of the $\mathrm{SnS}$ electrode without the $\mathrm{SiO}_{2}$ layer became white under visible light irradiation, while its color was relatively retained by $\mathrm{SiO}_{2}$ coating.

We also evaluated the oxygen production properties of SnS and $\mathrm{SnS}_{2}$ in the presence of aqueous $\mathrm{Ag}^{+}$as a reductive sacrificial agent; however, neither compound exhibited photocatalytic activity for water oxidation under these conditions. The valence band of $\mathrm{SnS}$ is not deep enough to produce oxygen, and $\mathrm{SnS}_{2}$ was corroded by visible light-induced oxidation. Based on these results, we can conclude that $\mathrm{SnS}$ is a promising candidate for a visible-light sensitive photocathode for hydrogen production and can be combined with a photoanode to construct a $Z$-scheme system for efficient overall water splitting.

In conclusion, density functional theory calculations were used to determine the electronic structure of the tin sulfides $\mathrm{SnS}$ and $\mathrm{SnS}_{2}$ and suggested that $\mathrm{SnS}$ is a potentially suitable photocathode for solar hydrogen production. Specifically, SnS has sufficiently low electron affinity to produce hydrogen from water and also has a small ionization potential, allowing for the absorption of a wide range of visible light. We experimentally synthesized SnS QDs using the SILAR method and demonstrated that this material could be used for efficient photocatalytic hydrogen production when the bandgap of SnS was wider than that of the bulk material. Because SnS is composed of safe and abundant elements, and SnS QDs can be synthesized using a facile wet chemical method that is applicable for mass production, we anticipate that the SnS QDs described here can be applied as photocathodes for solar hydrogen production.

We thank Mr K. Hori and Mr J. Koki at the Center for Advanced Materials Analysis of the Tokyo Institute of Technology for performing the TEM and SEM observations. We also appreciate the help of RIKEN KEIKI Co., Ltd for the UPS measurements and Ms G. Yin at the Tokyo Institute of Technology for the XPS analysis. We acknowledge Mr G. Newton for the critical reading of the manuscript. This research was supported by JST-PRESTO and JST-ACT-C programs, and JSPS KAKENHI Grant Number 26410234.

\section{Notes and references}

1 A. Fujishima and K. Honda, Nature, 1972, 238, 37-38.

2 K. Maeda, K. Teramura, D. Lu, T. Takata, N. Saito, Y. Inoue and K. Domen, Nature, 2006, 440, 295.

3 Q. Wang, T. Hisatomi, Q. Jia, H. Tokudome, M. Zhong, C. Wang, Z. Pan, T. Takata, M. Nakabayashi, N. Shibata, Y. Li, I. D. Sharp, A. Kudo, T. Yamada and K. Domen, Nat. Mater., 2016, DOI: 10.1038/ nmat4589.

4 S. Narushima, M. Hiroki, K. Ueda, K.-I. Shimizu, T. Kamiya, M. Hirano and H. Hosono, Philos. Mag. Lett., 2004, 84, 665-671.

5 A. J. Hoffman, G. Mills, H. Yee and M. R. Hoffmann, J. Phys. Chem., 1992, 96, 5546-5552.

6 I. Tsuji, H. Kato and A. Kudo, Angew. Chem., 2005, 117, 3631-3634.

7 W. Septina, Gunawan, S. Ikeda, T. Harada, M. Higashi, R. Abe and M. Matsumura, J. Phys. Chem. C, 2015, 119, 8576-8583.

8 D. R. Baker and P. V. Kamat, Adv. Funct. Mater., 2009, 19, 805-811.

9 R. Vogel, P. Hoyer and H. Weller, J. Phys. Chem., 1994, 98, 3183-3188.

10 Y. Jin-nouchi, T. Hattori, Y. Sumida, M. Fujishima and H. Tada, ChemPhysChem, 2010, 11, 3592-3595.

11 Q. Shen, D. Arae and T. Toyoda, J. Photochem. Photobiol., A, 2004, 164, 75-80.

12 J. Y. Chang, J. M. Lin, L. F. Su and C. F. Chang, ACS Appl. Mater. Interfaces, 2013, 5, 8740-8752. 
13 N. Srinivasan, Y. Shiga, D. Atarashi, E. Sakai and M. Miyauchi, Appl. Catal., B, 2015, 179, 113-121.

14 A. Kondo, G. Yin, N. Srinivasan, D. Atarashi, E. Sakai and M. Miyauchi, Nanoscale, 2015, 7, 12510-12515.

15 H. Hosono, K. Hayashi, T. Kamiya, T. Atou and T. Susaki, Sci. Technol. Adv. Mater., 2011, 12, 034303.

16 H. Takahashi, K. Igawa, K. Arii, Y. Kamihara, M. Hirano and H. Hosono, Nature, 2008, 453, 376-378.

17 Y. Hirayama, Y. K. Takahashi, S. Hirosawa and K. Hono, Scr. Mater., 2015, 95, 70-72.

18 X. Wang, K. Maeda, A. Thomas, K. Takanabe, G. Xin, J. M. Carlsson, K. Domen and M. Antonietti, Nat. Mater., 2009, 8, 76-80.

19 C. Huang, C. Chen, M. Zhang, L. Lin, X. Ye, S. Lin, M. Antonietti and X. Wang, Nat. Commun., 2015, 6, 7698.

20 J. Zhang, Y. Chen and X. Wang, Energy Environ. Sci., 2015, 8, 3092-3108.

21 Y. Ogo, H. Hiramatsu, K. Nomura, H. Yanagi, T. Kamiya, M. Hirano and H. Hosono, Appl. Phys. Lett., 2008, 93, 032113.

22 L. A. Burton, D. Colombara, R. D. Abellon, F. C. Grozema, L. M. Peter, T. J. Savenije, G. Dennler and A. Walsh, Chem. Mater., 2013, 25, $4908-4916$.
23 L. A. Burton and A. Walsh, Appl. Phys. Lett., 2013, 102, 132111.

24 W. Albers, C. Haas, H. J. Vink and J. D. Wasscher, J. Appl. Phys., 1961, 32, 2220-2225.

25 Z. Zainal, M. Z. Hussein and A. Ghazali, Sol. Energy Mater. Sol. Cells, 1996, 40, 347-357.

26 A. Sanchez-Juarez and A. Ortíz, Semicond. Sci. Technol., 2002, 17, 931.

27 L. Amalraj, C. Sanjeeviraja and M. Jayachandran, J. Cryst. Growth, 2002, 234, 683-689.

28 G. Domingo, R. S. Itoga and C. R. Kannewurf, Phys. Rev., 1966, 143, 536-541.

29 M. Miyauchi, Chem. Phys. Lett., 2011, 514, 151-155.

30 M. Miyauchi, Y. Shiga, N. Srinivasan, D. Atarashi and E. Sakai, Mater. Chem. Phys., 2015, 160, 383-388.

31 Y. C. Zhang, J. Li, M. Zhang and D. D. Dionysiou, Environ. Sci. Technol., 2011, 45, 9324-9331.

32 D. Chen, G. Shen, K. Tang, S. Lei, H. Zheng and Y. Qian, J. Cryst. Growth, 2004, 260, 469-474.

33 K. Zhao, Z. Pan, I. Mora-Seró, E. Cánovas, H. Wang, Y. Song, X. Gong, J. Wang, M. Bonn, J. Bisquert and X. Zhong, J. Am. Chem. Soc., 2015, 137, 5602-5609. 